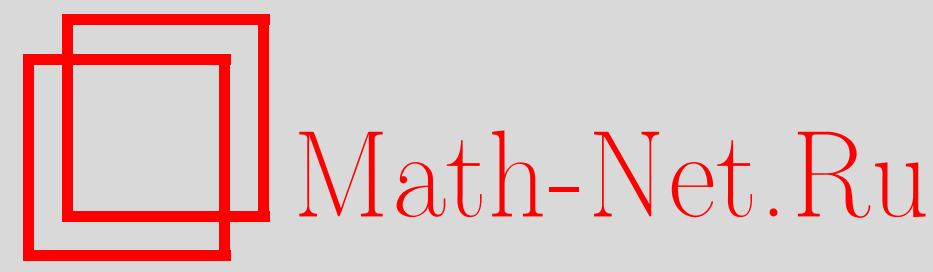

Н. В. Афанасьева, А. Ф. Тедеев, Теоремы типа Фуджиты для квазилинейных параболических уравнений в случае медленно стремящихся к нулю начальных данных, Матем. сб., 2004, том 195, номер 4, 3-22

DOI: https://doi.org/10.4213/sm812

Использование Общероссийского математического портала Math-Net.Ru подразумевает, что вы прочитали и согласны с пользовательским соглашением http://www . mathnet.ru/rus/agreement

Параметры загрузки:

IP: 18.234 .197 .8

26 апреля 2023 г., 16:55:44 
УДК 517.946

Н.В. Афанасьева, А.Ф. Тедеев

\section{Теоремы типа Фуджиты для квазилинейных параболических уравнений в случае медленно стремящихся к нулю начальных данных}

В настоящей работе рассматривается задача Коши для параболического уравнения с двойной нелинейностью следующего вида:

$$
u_{t}=\operatorname{div}\left(u^{\alpha}|D u|^{m-1} D u\right)+u^{p},
$$

где $0<m+\alpha \leqslant 1$. Устанавливаются существование и несуществование в целом по времени решений этой задачи для начальных данных, медленно стремящихся к нулю.

Библиография: 22 названия.

\section{§1. Введение}

Рассматривается следуюшая задача Коши:

$$
\begin{gathered}
u_{t}=\operatorname{div}\left(u^{\alpha}|D u|^{m-1} D u\right)+u^{p}, \\
(x, t) \in Q_{T}=\mathbb{R}^{N} \times(0, T), \quad T>0, \quad N \geqslant 1, \\
u(x, 0)=u_{0}(x), \quad x \in \mathbb{R}^{N} .
\end{gathered}
$$

Здесь предполагается, что $m+\alpha \leqslant 1, m>0, m+\alpha>\max \{0,1-(m+1) / N\}$, $p>1, u_{0}(x)$ - неотрицательная измеримая функция из класса $L_{1, \operatorname{loc}}\left(\mathbb{R}^{N}\right)$. При $m+\alpha-1<0$ уравнение (1.1) без источника относится к уравнениям быстрой диффузии, и при $m=1$ оно возникает в физике плазмы [1]. Характерной особенностью этого класса уравнений является отсутствие свойства конечной скорости распространения возмушений. Вопросам разрешимости и гладкости решений начально-краевых задач посвяшены работы [2]-[4].

Основной целью данной работы является установление условий сушествования и несуществования в целом по времени решений задачи (1.1), (1.2) для некоторых классов начальных функций, вообще говоря, не принадлежащих $L_{1}\left(\mathbb{R}^{N}\right)$.

Хорошо известно (см., например, [5]), что даже в простейшем случае $m=1$, $\alpha=0$ сушествуют решения (1.1), которые неограниченно растут за конечноевремя ("взрьваются"), т.е. существует $T>0$ такое, что

$$
\|u(\cdot, t)\|_{\infty, \mathbb{R}^{N}} \rightarrow \infty, \quad t \rightarrow T<\infty
$$

Работа выполнена при поддержке фонда INTAS (грант № 97-30551) и ДФФД Украины (грант № 01.07/00252). 
В работе [6] было показано, что если $p<p^{*}=1+2 / N$, то любое неотрицательное решение задачи (1.1), (1.2) "взрьвается" за конечное время. Если же $p>p^{*}$, то для начальных функций, достаточно малых в определенном смысле, сушествует решение в целом по времени. Позже в работе [7] было установлено, что $p=p^{*}$ включается в случай режима с обострением.

Результаты такого сорта принято называть теоремами типа Фуджиты и Фуджиты-Хаякавы соответственно, а число $p^{*}$ называется критическим показателем. Оказалось, что $p^{*}$ существенно зависит от класса рассматриваемых задач. Например, если в (1.1) $\alpha=0, m>1$, то $p^{*}=m+(m+1) / N$, и $p^{*}=\alpha+1+2 / N$, если $\alpha>0, m=1$ (см. [8]). В общем случае, если $m+\alpha-1>0$, то $p^{*}=m+\alpha+(m+1) / N$ (см. [9]-[11]). Отметим также работу [12], где рассмотрен случай $m+\alpha-1 \leqslant 0$. Во всех указанных работах предполагалось, что $u_{0} \in L_{1}\left(\mathbb{R}^{N}\right)$. Результаты работы [13] показали, что $p^{*}$ сушественно зависит также от поведения начальных данных на бесконечности при условии, что $u_{0} \notin L_{1}\left(\mathbb{R}^{N}\right)$. Асимптотическое поведение решений в случае медленно убывающих начальных данных изучалось в работе [14]. Подробньй анализ результатов по теоремам типа Фуджиты и Фуджиты-Хаякавы можно найти в обзорных работах [9], [15], [16].

Прежде чем перейти к формулировкам основных результатов работы, введем некоторые понятия.

Пусть $h(s), s>0,-$ непрерывная неотрицательная неубывающая функция такая, что $h(s) \rightarrow \infty$ при $s \rightarrow \infty$. Кроме того, предположим, что функция $\mathscr{H}(s)=s^{m+1} h^{m+\alpha-1}(s)$ не убывает для всех $s>0$.

Пусть $B_{\rho}\left(x_{0}\right)=\left\{\left|x-x_{0}\right|<\rho\right\}-$ шар радиуса $\rho$ с центром в $x_{0} \in \mathbb{R}^{N}$. Предположим, что $u_{0}$ такова, что

$$
\left[u_{0}\right]=\sup _{x_{0} \in \mathbb{R}^{N}} \sup _{\rho>0} h(\rho) f_{B_{\rho}\left(x_{0}\right)} u_{0}(x) d x<\infty
$$

где

$$
\begin{gathered}
f_{B_{\rho}\left(x_{0}\right)} \cdot d x=\left|B_{\rho}\left(x_{0}\right)\right|^{-1} \int_{B_{\rho}\left(x_{0}\right)} \cdot d x, \\
\left|B_{\rho}\left(x_{0}\right)\right|=\operatorname{mes}_{N} B_{\rho}\left(x_{0}\right) .
\end{gathered}
$$

Введем теперь понятие обобщенного решения задачи (1.1), (1.2).

Будем говорить, что $u(x, t)$ является обобщеннылм решением задачи (1.1), (1.2) в $Q_{T}$, если $u(x, t) \geqslant 0$ почти всюду при $(x, t) \in Q_{T}$,

$$
\begin{gathered}
u \in L_{\infty, \mathrm{loc}}\left(Q_{T}\right) \cap C\left((0, T) ; L_{2, \mathrm{loc}}\left(\mathbb{R}^{N}\right)\right), \\
u^{\alpha}|D u|^{m+1} \in L_{1, \mathrm{loc}}\left(Q_{T}\right), \\
u^{p} \in L_{1, \mathrm{loc}}\left(Q_{T}\right),
\end{gathered}
$$

функция $u(x, t)$ удовлетворяет интегральному тождеству

$$
\int_{Q_{T}}\left(-u \eta_{t}+u^{\alpha}|D u|^{m-1} D u D \eta\right) d x d t=\int_{Q_{T}} u^{p} \eta d x d t
$$


для любой функции $\eta(x, t) \in C_{0}^{1}\left(Q_{T}\right)$. И кроме того,

$$
u(\cdot, t) \rightarrow u_{0} \text { при } t \rightarrow 0 \text { в } L_{1, \operatorname{loc}}\left(\mathbb{R}^{N}\right) .
$$

Понятие суб- и суперрешения (1.1), (1.2) определяется стандартно, заменой в (1.4) знака равенства на знаки " $\leqslant$ " и " $\geqslant$ " соответственно. В дальнейшем обобщенное решение будем назьвать просто решением.

Пусть $\mathscr{R}(\tau)$ - обратная к $\mathscr{H}(s)$ функция. Основньми результатами данной работы являются следующие теоремы.

TEOPEMA 1.1. Пусmb

$$
\int_{1}^{\infty} \frac{d \tau}{h^{p-1}(\mathscr{R}(\tau))}<\infty
$$

Тогда существует решение $u(x, t)$ задачи (1.1), (1.2), определенное для всех $t>0$, при условии, что

$$
\left\|u_{0}\right\|_{L_{q}\left(\mathbb{R}^{N}\right)}+\left[u_{0}\right] \leqslant \delta,
$$

где $q>N(p-m-\alpha) /(m+1)$ u $\delta=\delta(N, m, \alpha)$ - достаточно малое число. Более того, найдется такая положительная постоянная $\gamma_{0}$, что для всех $t>0$ справедлива оценка

$$
\|u(\cdot, t)\|_{\infty, \mathbb{R}^{N}} \leqslant \gamma_{0} \frac{\left[u_{0}\right]}{h\left(\mathscr{R}\left(\left[u_{0}\right]^{m+\alpha-1} t\right)\right)} .
$$

ТЕОРема 1.2. Пусть для функции $h(s)$ выполняется следующее: существуют $x_{0} \in \mathbb{R}^{N}$ и положстельные числа $\varepsilon_{0}, \theta_{0}<m+\alpha$ такие, что

$$
h(\lambda s) \leqslant \lambda^{\varepsilon_{0}} h(s), \quad f_{B_{\rho}\left(x_{0}\right)} u_{0}^{1-\theta}(x) d x \geqslant \gamma_{1} h^{-(1-\theta)}(\rho)
$$

для всех $s>0, \lambda>1, \rho>0,0<\theta<\theta_{0}$ и некоторой постоянной $\gamma_{1}$.

Кроме того, пусть

$$
\frac{h(\rho)}{\rho^{\frac{m+1}{p-m-\alpha}}} \rightarrow 0, \quad \rho \rightarrow \infty .
$$

Тогда любое решение задачи (1.1), (1.2) “взрьвается” за конечное время.

ЗАмечАниЕ 1.1. Теорема 1.1 была доказана для случая $m+\alpha-1>0$ в работе [17].

ЗАмЕчАнИЕ 1.2. Если начальная функция имеет вид $u_{0}=a|x|^{-\beta}$, где $0<$ $\beta<N$ и $a>0$ - некоторое достаточно малое число, то очевидно, что мы можем положить $h(\rho)=\rho^{\beta}$. Тогда из теорем 1.1 и 1.2 следует, что $p^{*}=m+\alpha+(m+1) / \beta$, и при $m=1$ данный результат совпадает с результатом в [13].

ЗАмечАниЕ 1.3. Второе из неравенств (1.8) в теореме 1.2 выполняется, например, если $u_{0} \geqslant c h^{-1}(|x|)$ с некоторой постоянной $c>0$.

При доказательстве теорем 1.1, 1.2 используются и развиваются энергетические подходы работ [2], [10], [18]-[20].

Авторы искренне благодарят рецензента за ряд ценных замечаний. 


\section{§2. Доказательство теоремы 1.1}

Рассмотрим последовательность $u_{n}(x, t), n \geqslant 1$, решений следуюшей начально-краевой задачи Коши-Дирихле:

$$
\begin{gathered}
u_{t}-\operatorname{div}\left(u^{\alpha}|D u|^{m-1} D u\right)=\min \left\{u^{p}, n\right\}, \\
(x, t) \in B_{n} \times(0, \infty), \\
u=0 \text { на } \partial B_{n} \times(0, \infty), \\
u(x, 0)=u_{0 n}, \quad x \in B_{n},
\end{gathered}
$$

где $B_{n}=B_{\rho_{n}}(0), \rho_{n} \rightarrow \infty, n \rightarrow \infty$ и $u_{0 n} \in C_{0}^{\infty}\left(B_{n}\right)$ такие, что $u_{0 n} \rightarrow u_{0}$ в $L_{1, \text { loc }}\left(\mathbb{R}^{N}\right) \cap L_{q}\left(\mathbb{R}^{N}\right)$. Продолжив $u_{n}(x, t)$ нулем вне $B_{n} \times(0, \infty)$, мы тем самым определим его во всем $\mathbb{R}^{N} \times(0, \infty)$. Из результатов работы [4] следует, что задача (2.1)-(2.3) глобально разрешима при всяком фиксированном $n$. Всюду в дальнейшем мы опустим индекс $n$, обозначив $u_{n}$ через $u$.

Пусть

$$
\langle u\rangle_{t}=\sup _{0<\tau<t} \sup _{x_{0} \in \mathbb{R}^{N}} \sup _{\rho>\mathscr{R}(C t)} h(\rho) f_{B_{\rho}\left(x_{0}\right)} u(\cdot, \tau) d x .
$$

Нам потребуются следуюшие леммы.

ЛЕмма 2.1. Пусть и $(x, t)$ является непрерывным решением уравнения (2.1) и $T>0$ такое, что неравенство

$$
\frac{t}{\mathscr{R}^{m+1}(C t)}\|u(\cdot, t)\|_{\infty, \mathbb{R}^{N}}^{m+\alpha-1}+t\|u(\cdot, t)\|_{\infty, \mathbb{R}^{N}}^{p-1}<1
$$

выполнено для всех $0<t<T$, где $C=C_{0}\left[u_{0}\right]^{m+\alpha-1}, C_{0}>0$ - постоянная, зависящая лишь от $N, m, \alpha, p$.

Тогда для всех $0<t<T$ найдется постоянная $\gamma=\gamma(N, m, \alpha, p)$ такая, что верно неравенство

$$
\|u(\cdot, t)\|_{\infty, \mathbb{R}^{N}} \leqslant \gamma \frac{C^{\frac{N}{K}}\langle u\rangle_{t}^{\frac{m+1}{K}}}{h(\mathscr{R}(C t))},
$$

где $K=N(m+\alpha-1)+m+1$.

Здесь и далее через $\gamma$ будем обозначать постоянные, зависящие лишш от $N, m$, $\alpha, p$.

ЛЕмма 2.2. Пусть выполнены условия леммы 2.1. Тогда для всех $\rho>\mathscr{R}(C t)$, $x_{0} \in \mathbb{R}^{N}$ верны следующие неравенства:

1) если $\alpha+m<1, m o$

$$
\begin{aligned}
& \frac{h(\rho)}{\rho^{N+1}} \int_{0}^{t} \int_{B_{\rho}\left(x_{0}\right)} u^{\alpha}|D u|^{m} d x d \tau \\
& \quad \leqslant \gamma\left(C^{-\frac{1}{m+1}}\langle u\rangle_{t}^{1+\frac{m+\alpha-1}{m+1}}+C^{-\frac{1}{1-m-\alpha}}\right)
\end{aligned}
$$

2) если $\alpha+m=1, m o$

$$
\frac{h(\rho)}{\rho^{N+1}} \int_{0}^{t} \int_{B_{\rho}\left(x_{0}\right)} u^{\alpha}|D u|^{m} d x d \tau \leqslant \gamma C^{-\frac{m}{(m+1)^{2}}}\langle u\rangle_{t} .
$$


ДОКАЗАТЕЛЬСТВО ЛЕМмЫ 2.1. В силу условий леммы справедлива следующая оценка:

$$
\|u(\cdot, t)\|_{\infty, B_{\rho}\left(x_{0}\right)} \leqslant \gamma t^{-\frac{N}{K}}\left(f_{0}^{t} \int_{B_{2 \rho}\left(x_{0}\right)} u(\cdot, \tau) d x d \tau\right)^{\frac{m+1}{K}}
$$

для всех $0<t<T$ и $\rho \geqslant \mathscr{R}(C t)$.

Доказательство оценки (2.8) проводится по той же схеме, что и в работах [2], [21]. Мы приведем его ниже, после доказательства теоремы 1.1.

Положим в $(2.8) \rho=\mathscr{R}(C t)$. Поскольку из определения функции $\mathscr{R}(\tau)$ следует, что $t=C^{-1} \mathscr{R}^{m+1}(C t) h^{m+\alpha-1}(\mathscr{R}(C t))$, то из $(2.8)$, воспользовавшись определением $\langle u\rangle_{t}$, получаем

$$
\begin{aligned}
\|u(\cdot, t)\|_{\infty, B_{\mathscr{R}(C t)}\left(x_{0}\right)} & \leqslant \gamma \frac{t^{-\frac{N}{K} \mathscr{R}} \frac{(m+1) N}{K}(C t)}{h^{\frac{m+1}{K}}(\mathscr{R}(C t))}\langle u\rangle_{t}^{\frac{m+1}{K}} \\
& \leqslant \gamma \frac{C^{\frac{N}{K}}\langle u\rangle_{t}^{\frac{m+1}{K}}}{h(\mathscr{R}(C t))} .
\end{aligned}
$$

Наконец, так как $x_{0} \in \mathbb{R}^{N}$ в (2.9) произвольно, то мы приходим к требуемой оценке (2.5). Лемма 2.1 доказана.

ДоКАЗАТЕЛЬСтво ЛЕммы 2.2. Пусть $v=u^{\frac{\alpha+m}{m}}$ и $\zeta(x)$-гладкая срезающая функция шара $B_{2 \rho} \equiv B_{2 \rho}\left(x_{0}\right)$ такая, что $0 \leqslant \zeta \leqslant 1, \zeta=1$ на $B_{\rho}, \zeta=0$ вне $B_{2 \rho}$ и $|D \zeta| \leqslant \gamma / \rho$.

Далее рассмотрим отдельно два случая. Случай $\alpha+m<1$. По неравенству Гёльдера

$$
\begin{aligned}
\int_{0}^{t} \int_{B_{2 \rho}} \zeta^{m}|D v|^{m} d x d \tau \leqslant \gamma\left(\int_{0}^{t} \int_{B_{2 \rho}}(t-\tau)^{\frac{\theta(m+1)}{m}}\right. \\
\left.\quad \times|D v|^{m+1}(v+\varepsilon)^{-\frac{\lambda(m+1)}{m}} \zeta^{m+1} d x d \tau\right)^{\frac{m}{m+1}} \\
\quad \times\left(\int_{0}^{t} \int_{B_{2 \rho}}(t-\tau)^{-\theta(m+1)}(v+\varepsilon)^{\lambda(m+1)} d x d \tau\right)^{\frac{1}{m+1}} \\
\equiv I_{1}^{\frac{m}{m+1}} I_{2}^{\frac{1}{m+1}}
\end{aligned}
$$

где числа $\theta>0, \lambda>0, \varepsilon>0$ будут выбраны позже.

Пусть

$$
\varphi=(t-\tau)^{\frac{\theta(m+1)}{m}}(v+\varepsilon)^{1-\frac{\lambda(m+1)}{m}} \zeta^{m+1} .
$$

Тогда, умножая обе части (2.1) на $\varphi$ и интегрируя по $B_{2 \rho} \times(0, t)$, получаем

$$
\begin{aligned}
0 \leqslant & \int_{0}^{t} \int_{B_{2 \rho}}\left(v^{\frac{m}{\alpha+m}}\right)_{\tau}(v+\varepsilon)^{1-\frac{\lambda(m+1)}{m}}(t-\tau)^{\frac{\theta(m+1)}{m}} \zeta^{m+1} d x d \tau \\
& +\left(\frac{m}{\alpha+m}\right)^{m} \int_{0}^{t} \int_{B_{2 \rho}}|D v|^{m-1} D v D \varphi d x d \tau \equiv I_{3}+\left(\frac{m}{\alpha+m}\right)^{m} I_{4} .
\end{aligned}
$$


Оценим слагаемое $I_{3}$ :

$$
\begin{aligned}
I_{3} \leqslant & \frac{\theta(m+1)}{m} \int_{0}^{t} \int_{B_{2 \rho}}(v+\varepsilon)^{1-\frac{\lambda(m+1)}{m}+\frac{m}{\alpha+m}} \\
& \times(t-\tau)^{\frac{\theta(m+1)}{m}-1} \zeta^{m+1} d x d \tau-\frac{(m-\lambda(m+1))(\alpha+m)}{m^{2}+(m-\lambda(m+1))(\alpha+m)} \\
& \times \int_{0}^{t} \int_{B_{2 \rho}}\left((v+\varepsilon)^{1-\frac{\lambda(m+1)}{m}+\frac{m}{\alpha+m}}\right)_{\tau}(t-\tau)^{\frac{\theta(m+1)}{m}} \zeta^{m+1} d x d \tau .
\end{aligned}
$$

Подберем $\lambda$ так, чтобы

$$
\frac{(m-\lambda(m+1))(\alpha+m)}{m^{2}+(m-\lambda(m+1))(\alpha+m)}<0 .
$$

Это будет выполнено, например, если

$$
\lambda=\frac{m(\alpha+2 m)}{(m+1)^{2}(\alpha+m)} .
$$

Тогда, интегрируя по частям второе слагаемое в правой части (2.12), получаем

$$
\begin{aligned}
I_{3} & \leqslant \gamma \int_{0}^{t} \int_{B_{2 \rho}}(v+\varepsilon)^{\frac{m(\alpha+2 m)}{(m+1)(\alpha+m)}}(t-\tau)^{\frac{\theta(m+1)}{m}-1} \zeta^{m+1} d x d \tau \\
& \leqslant \gamma \int_{0}^{t} \int_{B_{2 \rho}}(v+\varepsilon)^{\frac{m(\alpha+2 m)}{(m+1)(\alpha+m)}}(t-\tau)^{\frac{\theta(m+1)}{m}-1} d x d \tau \equiv \gamma I_{5} .
\end{aligned}
$$

Далее имеем

$$
\begin{aligned}
& I_{4} \leqslant- \frac{m(1-m-\alpha)}{(m+1)(\alpha+m)} I_{1}+(m+1) \int_{0}^{t} \int_{B_{2 \rho}}|D v|^{m}(v+\varepsilon)^{1-\frac{\alpha+2 m}{(m+1)(\alpha+m)}} \\
& \times \zeta^{m}|D \zeta|(t-\tau)^{\frac{\theta(m+1)}{m}} d x d \tau \equiv-\gamma I_{1}+(m+1) I_{6} .
\end{aligned}
$$

Применяя неравенство Юнга, находим:

$$
\begin{aligned}
I_{6} & \leqslant \frac{m}{m+1} \sigma^{\frac{m+1}{m}} I_{1} \\
& +\frac{\sigma^{-(m+1)}}{m+1} \frac{t}{\rho^{m+1}} \int_{0}^{t} \int_{B_{2 \rho}}(v+\varepsilon)^{\frac{m(\alpha+2 m)}{(m+1)(\alpha+m)}-\frac{m(1-m-\alpha)}{\alpha+m}}(t-\tau)^{\frac{\theta(m+1)}{m}-1} d x d \tau .
\end{aligned}
$$

Таким образом, при достаточно малом $\sigma>0$ из (2.14) находим

$$
I_{4} \leqslant-\gamma I_{1}+\gamma \frac{t}{\rho^{m+1}} \frac{I_{5}}{\varepsilon^{\frac{m}{m+\alpha}}(1-m-\alpha)} .
$$

Следовательно, из последнего неравенства и оценок $(2.11),(2.13)$ имеем

$$
I_{1} \leqslant \gamma\left(1+\frac{t}{\rho^{m+1} \varepsilon^{\frac{m}{m+\alpha}}(1-m-\alpha)}\right) \int_{0}^{t} \int_{B_{2 \rho}}(v+\varepsilon)^{\frac{m(\alpha+2 m)}{(m+1)(\alpha+m)}}(t-\tau)^{\frac{\theta(m+1)}{m}}-1 d x d \tau .
$$


Пусть

$$
\theta=\frac{m}{(m+1)^{2}}, \quad \varepsilon=\left(\frac{t}{\rho^{m+1}}\right)^{\frac{\alpha+m}{m(1-m-\alpha)}}
$$

Тогда из $(2.15)$

$$
I_{1} \leqslant \gamma I_{2}
$$

Отсюда и из (2.10) следует

$$
\begin{aligned}
& \frac{1}{\rho} \int_{0}^{t} \int_{B_{2 \rho}} u^{\alpha}|D u|^{m} d x d \tau \leqslant \gamma \frac{t^{\frac{1}{m+1}}}{\rho} \sup _{0<\tau<t} \int_{B_{2 \rho}} u^{\frac{\alpha+2 m}{m+1}} d x \\
& \quad+\gamma\left(\frac{t}{\rho^{m+1}}\right)^{\frac{\alpha+2 m}{(m+1)(1-m-\alpha)}} t^{\frac{1}{m+1}} \rho^{N-1} .
\end{aligned}
$$

Наконец, применяя в (2.16) неравенство Юнга, получаем требуемую оценку (2.6).

Перейдем к рассмотрению случая $m+\alpha=1$. Пусть теперь $\lambda>0$ - некоторая постоянная, которая будет выбрана позже. Применяя неравенство Гёльдера, получаем

$$
\begin{gathered}
\int_{0}^{t} \int_{B_{2 \rho} \rho} \zeta^{m}|D v|^{m} d x d \tau \leqslant \gamma\left(\int_{0}^{t} \int_{B_{2 \rho}}|D v|^{m+1} \zeta^{m+1} v^{\lambda m-1} \tau^{\frac{1}{m+1}} d x d \tau\right)^{\frac{m}{m+1}} \\
\times\left(\int_{0}^{t} \int_{B_{2} \rho} v^{(1-\lambda m) m} \tau^{-\frac{m}{m+1}} d x d \tau\right)^{\frac{1}{m+1}} \equiv \gamma J_{1}^{\frac{m}{m+1}} J_{2}^{\frac{1}{m+1}}
\end{gathered}
$$

Пусть

$$
\varphi(x, t)=t^{\frac{1}{m+1}} v^{\lambda m}(x, t) \zeta^{m+1}(x),
$$

где функция $\zeta$ та же, что и в предыдущем случае. Снова, умножая обе части (2.1) на $\varphi$ и интегрируя по $Q_{T}$, получаем

$$
\begin{aligned}
& \int_{0}^{t} \int_{B_{2 \rho} \rho}\left(v^{m}\right)_{\tau} \tau^{\frac{1}{m+1}} v^{\lambda m} \zeta^{m+1} d x d \tau+m^{m} \int_{0}^{t} \int_{B_{2 \rho}}|D v|^{m-1} D v D \varphi d x d \tau \\
& \quad \leqslant \gamma \int_{0}^{t} \int_{B_{2 \rho}} v^{m p+\lambda m} \tau^{\frac{1}{m+1}} \zeta^{m+1} d x d \tau
\end{aligned}
$$

Первое и второе слагаемые в левой части (2.18) оцениваются снизу через

$$
-\frac{1}{(1+\lambda)(m+1)} \int_{0}^{t} \int_{B_{2 \rho}} v^{m(1+\lambda)} \tau^{-\frac{m}{m+1}} d x d \tau \equiv-\frac{1}{(1+\lambda)(m+1)} J_{3}
$$

И

$$
\begin{aligned}
& \frac{m \lambda}{2} \int_{0}^{t} \int_{B_{2 \rho}}|D v|^{m+1} v^{\lambda m-1} \tau^{\frac{1}{m+1}} \zeta^{m+1} d x d \tau \\
& \quad-\gamma \frac{t}{\rho^{m+1}} \int_{0}^{t} \int_{B_{2 \rho}} v^{m(1+\lambda)} \tau^{-\frac{m}{m+1}} d x d \tau \equiv \frac{m \lambda}{2} J_{1}-\gamma \frac{t}{\rho^{m+1}} J_{3}
\end{aligned}
$$

соответственно. Здесь мы воспользовались также неравенством Юнга. 
Оценим теперь правую часть в (2.18) сверху, используя условие (2.4), следующей величиной:

$$
\int_{0}^{t} \tau\left\|v^{m}(\cdot, \tau)\right\|_{\infty, \mathbb{R}^{N}}^{p-1} \int_{B_{2 \rho}} \tau^{-\frac{m}{m+1}} v^{m(\lambda+1)} d x d \tau \leqslant J_{3} .
$$

Таким образом, получаем

$$
J_{1} \leqslant \gamma(N, m, \alpha, p, \lambda)\left(1+\frac{t}{\rho^{m+1}}\right) J_{3} .
$$

Поскольку в силу условия (2.4) $t / \rho^{m+1}<1$ при $m+\alpha-1=0$, то

$$
J_{1} \leqslant \gamma(N, m, \alpha, p, \lambda) J_{3}
$$

Выберем $\lambda$ из условия $\lambda<\min \{1 / N, 1 /(N m)\}$, тогда $1-N \lambda>0$. Воспользовавшись теперь оценкой (2.8) для интеграла $J_{1}$, получаем следующее неравенство:

$$
\begin{aligned}
J_{1} & \leqslant \gamma\left(\sup _{0<\tau<t} \int_{B_{2 \rho}} v^{m} d x\right) \int_{0}^{t}\left\|v^{m}(\cdot, \tau)\right\|_{\infty, \mathbb{R}^{N}}^{\lambda} \tau^{-\frac{m}{m+1}} d \tau \\
& \leqslant \gamma\left(\sup _{0<\tau<t} \int_{B_{2 \rho}} v^{m} d x\right)^{\lambda+1} \int_{0}^{t} \tau^{-\frac{m+N \lambda}{m+1}} d \tau=\gamma\left(\sup _{0<\tau<t} \int_{B_{2 \rho}} u d x\right)^{\lambda+1} t^{\frac{1-N \lambda}{m+1}} .
\end{aligned}
$$

Здесь при выводе (2.19) мы вновь учли, что $t / \rho^{m+1}<1$. Оценим $J_{2}$ следующим образом. Для любого $\mu>0$ имеем

$$
\begin{aligned}
J_{2} & =\int_{0}^{t} \int_{B_{2 \rho} \cap\{u<\mu\}} u^{1-\lambda m} \tau^{-\frac{m}{m+1}} d x d \tau+\int_{0}^{t} \int_{B_{2 \rho} \cap\{u \geqslant \mu\}} u^{1-\lambda m} \tau^{-\frac{m}{m+1}} d x d \tau \\
& \leqslant \gamma\left(\mu^{1-\lambda m} t^{\frac{1}{m+1}} \rho^{N}+\mu^{-\lambda m} t^{\frac{1}{m+1}} \sup _{0<\tau<t} \int_{B_{2 \rho}} u d x\right) .
\end{aligned}
$$

Выбирая $\mu=\sup _{0<\tau<t} f_{B_{2 \rho}} u(\cdot, \tau) d x$, получаем

$$
J_{2} \leqslant \gamma t^{\frac{1}{m+1}} \rho^{N \lambda m}\left(\sup _{0<\tau<t} \int_{B_{2 \rho}} u(\cdot, \tau) d x\right)^{1-\lambda m} .
$$

Наконец, из (2.17)-(2.20) приходим к оценке

$$
\frac{1}{\rho} \int_{0}^{t} \int_{B_{\rho}} u^{\alpha}|D u|^{m} d x d \tau \leqslant \gamma\left(\frac{t}{\rho^{m+1}}\right)^{\frac{m}{(m+1)^{2}}} \sup _{0<\tau<t} \int_{B_{2 \rho}} u(\cdot, \tau) d x
$$

Умножая (2.16) и (2.21) на $h(\rho) \rho^{-N}$ и используя, что $s^{m+1} h^{m+\alpha-1}(s)-$ неубывающая функция, приходим к требуемому утверждению. Лемма 2.2 доказана.

Продолжим доказательство теоремы 1.1. Для доказательства глобальной разрешимости задачи $(1.1),(1.2)$ достаточно показать, что условие (2.4) выполнено 
для всех $t>0$ с константой $C$, зависяшей лишш от $N, m, \alpha, p,\left[u_{0}\right]$, но не зависящей от $n$.

Заметим, что первое слагаемое в (2.4) можно считать меньше $\frac{1}{2}$. В противном случае при $0<\alpha+m<1$ оценка (1.7) очевидна, а при $\alpha+m=1$ из определения функции $\mathscr{R}(t)$ получим

$$
\frac{t}{\mathscr{R}^{m+1}(C t)}=C^{-1} \leqslant \frac{1}{2}
$$

при достаточно большом $C$.

Покажем сначала, что

$$
t\|u(\cdot, t)\|_{\infty, \mathbb{R}^{N}}^{p-1} \leqslant \frac{1}{2} \quad \forall t \in\left(0, T^{\prime}\right)
$$

где $T^{\prime}>1$ при достаточно малой $\left\|u_{0}\right\|_{q, \mathbb{R}^{N}}$.

Рассуждая так же, как в [10], [18], положим

$$
T_{1}=\sup \{t>0:(2.22) \text { верно }\}
$$

Обе части (2.1) умножим на $u^{q-1}$, затем проинтегрируем по $Q_{T}$. В результате получим

$$
\int_{\mathbb{R}^{N}} u^{q}(\cdot, t) d x \leqslant \int_{\mathbb{R}^{N}} u_{0}^{q} d x+q \int_{0}^{t} \int_{\mathbb{R}^{N}} u^{p+q-1} d x d \tau .
$$

Воспользуемся оценкой

$$
\|u(\cdot, t)\|_{\infty, \mathbb{R}^{N}} \leqslant \gamma t^{-\frac{N}{K_{q}}}\left(\sup _{0<\tau<t} \int_{\mathbb{R}^{N}} u^{q}(\cdot, \tau) d x\right)^{\frac{m+1}{K_{q}}}
$$

где $K_{q}=N(m+\alpha-1)+q(m+1)$, доказательство которой мы приведем ниже. Тогда

$$
\int_{0}^{t} \int_{\mathbb{R}^{N}} u^{p+q-1} d x d \tau \leqslant \gamma \Psi(t) \Phi_{q}(t)
$$

где

$$
\Phi_{q}(t)=\sup _{0<\tau<t} \int_{\mathbb{R}^{N}} u^{q}(\cdot, \tau) d x, \quad \Psi(t)=t^{1-\frac{N(p-1)}{K_{q}}} \Phi_{q}^{\frac{(m+1)(p-1)}{K_{q}}}(t),
$$

что имеет смысл, если $q>N(p-m-\alpha) /(m+1)$.

Пусть $T_{0}=\sup \{t>0: \Psi(t) \leqslant \varepsilon\}$, где $\varepsilon>0-$ достаточно малое число. Таким образом, из неравенств $(2.23)$ и $(2.25)$ имеем

$$
\Phi_{q}(t) \leqslant \gamma\left\|u_{0}\right\|_{q, \mathbb{R}^{N}}^{q}, \quad 0<t<\min \left\{T_{0}, T_{1}\right\}
$$

Определим $T_{0}^{\prime}$ из равенства

$$
\gamma\left(T_{0}^{\prime}\right)^{1-\frac{N(p-1)}{K_{q}}}\left\|u_{0}\right\|_{q, \mathbb{R}^{N}}^{\frac{q(p-1)(m+1)}{K_{q}}}=\varepsilon
$$


Теперь, выбирая $\delta$ в условии (1.6) достаточно малым, можем считать, что $T_{0} \geqslant$ $T_{0}^{\prime}>1$. Если же $0<t<\min \left\{T_{0}^{\prime}, T_{1}\right\}$, то из $(2.24)$ и $(2.26)$

$$
t\|u(\cdot, t)\|_{\infty, \mathbb{R}^{N}}^{p-1} \leqslant \gamma t^{1-\frac{N(p-1)}{K_{q}}}\left\|u_{0}\right\|_{q, \mathbb{R}^{N}}^{\frac{q(p-1)(m+1)}{K_{q}}} \leqslant \frac{1}{4} .
$$

Отсюда следует, что $T_{1}>T_{0}^{\prime}>1$. И, значит, мы можем положить $T^{\prime}=T_{0}^{\prime}>1$.

Пусть $\zeta(x)$ - стандартная срезаюшая функция шара $B_{\rho}=B_{\rho}\left(x_{0}\right), x_{0}-$ произвольная точка $\mathbb{R}^{N}$. Умножая обе части $(2.1)$ на $\zeta(x)$ и интегрируя по $Q_{T}$, где $t \in\left(0, T^{\prime}\right)$, получаем

$$
\int_{B_{\rho / 2}} u(x, t) d x \leqslant \int_{B_{\rho}} u_{0} d x+\frac{\gamma}{\rho} \int_{0}^{t} \int_{B_{\rho}} u^{\alpha}|D u|^{m} d x d \tau+\int_{0}^{t} \int_{B_{\rho}} u^{p} d x d \tau .
$$

Рассмотрим два случая.

Пусть сначала $m+\alpha-1<0$. Тогда, умножая обе части $(2.28)$ на $h(\rho) \rho^{-N}$ и пользуясь леммой 2.2 , получаем

$$
\begin{aligned}
h(\rho) f_{B_{\rho / 2}} u(x, t) d x \leqslant & {\left[u_{0}\right]+\gamma C_{0}^{-\frac{1}{m+1}}\left[u_{0}\right]^{\frac{m+\alpha-1}{m+1}}\langle u\rangle_{t}^{1+\frac{m+\alpha-1}{m+1}} } \\
& +\gamma C_{0}^{-\frac{1}{1-m-\alpha}}\left[u_{0}\right]+\langle u\rangle_{t} \int_{0}^{t}\|u(\cdot, \tau)\|_{\infty, \mathbb{R}^{N}}^{p-1} d \tau .
\end{aligned}
$$

Поскольку $0<t<T^{\prime}$, то

$$
\int_{0}^{t}\|u(\cdot, \tau)\|_{\infty, \mathbb{R}^{N}}^{p-1} d \tau \leqslant t^{1-\frac{N(p-1)}{K_{q}}}\left\|u_{0}\right\|_{q, \mathbb{R}^{N}}^{\frac{q(p-1)(m+1)}{K_{q}}} \leqslant \varepsilon .
$$

Введем $t_{1}=\sup \left\{t>0:\langle u\rangle_{t} \leqslant 4 \gamma_{1}\left[u_{0}\right]\right\}$.

Тогда из (2.29) и (2.30) следует, что

$$
\begin{aligned}
& h(\rho) f_{B_{\rho / 2}} u(x, t) d x \leqslant\left[u_{0}\right]+\gamma C_{0}^{-\frac{1}{m+1}}\left(4 \gamma_{1}\right)^{1+\frac{m+\alpha-1}{m+1}}\left[u_{0}\right] \\
& \quad+\gamma C_{0}^{-\frac{1}{1-m-\alpha}}\left[u_{0}\right]+\varepsilon\langle u\rangle_{t}, \quad t \in\left(0, \min \left\{t_{1}, T^{\prime}\right\}\right) .
\end{aligned}
$$

Отсюда при достаточно большом $C_{0}=C_{0}(N, m, \alpha, p)$ и достаточно малом $\varepsilon=$ $\varepsilon(\delta)$ выводим

$$
\langle u\rangle_{t} \leqslant 2 \gamma_{1}\left[u_{0}\right] \quad \forall t \in\left(0, \min \left\{T_{0}^{\prime}, t_{1}\right\}\right)
$$

и, следовательно, $t_{1}>T^{\prime}$.

Покажем, наконец, что $T_{1}=\infty$. Для $t \in\left(1, T_{1}\right)$ так же, как при выводе (2.29), используя леммы 2.1 и 2.2 , имеем

$$
\begin{aligned}
h(\rho) & f_{B_{\rho / 2}} u(x, t) d x \\
& \leqslant\left[u_{0}\right]+\gamma\left(4 \gamma_{1}\right)^{\frac{(m+1)(p-1)}{K}}+1 C_{0}^{\frac{N(p-1)}{K}}\left[u_{0}\right]^{p} \int_{1}^{t} \frac{d \tau}{h^{p-1}\left(\mathscr{R}\left(C_{0}\left[u_{0}\right]^{m+\alpha-1} \tau\right)\right)}
\end{aligned}
$$


для всех $t \in\left(1, \min \left\{t_{1}, T_{1}\right\}\right)$. Делая замену переменных в последнем интеграле и учитывая, что $C_{0}\left[u_{0}\right]^{m+\alpha-1}>1$, получаем

$$
\langle u\rangle_{t} \leqslant \gamma\left[u_{0}\right]+\gamma C_{0}^{\frac{N(p-1)}{K}-1}\left[u_{0}\right]^{p-m-\alpha+1} D_{0},
$$

где

$$
D_{0}=\int_{1}^{\infty} \frac{d \tau}{h^{p-1}(\mathscr{R}(\tau))}<\infty .
$$

Следовательно, выбирая, если надо, $\delta$ достаточно малым, приходим к неравенству

$$
\langle u\rangle_{t} \leqslant 2 \gamma_{1}\left[u_{0}\right] \quad \forall t \in\left(0, \min \left\{t_{1}, T_{1}\right\}\right) .
$$

То есть $t_{1}>T_{1}$. Более того,

$$
\begin{aligned}
t\|u(\cdot, t)\|_{\infty, \mathbb{R}^{N}}^{p-1} & \leqslant \gamma(t-1)\left(\frac{\left[u_{0}\right]}{h\left(\mathscr{R}\left(\left[u_{0}\right]^{m+\alpha-1} t\right)\right)}\right)^{p-1}+\gamma\left(\frac{\left[u_{0}\right]}{h\left(\mathscr{R}\left(\left[u_{0}\right]^{m+\alpha-1} t\right)\right)}\right)^{p-1} \\
& \leqslant \gamma\left(\left[u_{0}\right]^{p-m-\alpha} D_{0}+\mathscr{R} \frac{m+1}{m+\alpha-1}\left(\left[u_{0}\right]^{m+\alpha-1}\right)^{p-1}\right) \leqslant \frac{1}{4}
\end{aligned}
$$

откуда выводим $T_{1}=\infty$. Теперь утверждение теоремы 1.1 для приближенного решения задачи следует из (2.32) и леммы 2.1. Поскольку мы получили оценки, которые не зависят от номера $n$ приближенного решения, то мы можем выделить из последовательности $u_{n}$ подпоследовательность, сходяшуюся к решению в $L_{1, \text { loc }}\left(\mathbb{R}^{N}\right)$, и для этого решения неравенство (1.7) будет выполнено.

Рассмотрим теперь случай $m+\alpha-1=0$. Рассуждая аналогично предыдушему случаю, для $t \in\left(0, T^{\prime}\right)$, где $T^{\prime}>1$, получаем, что

$$
\langle u\rangle_{t} \leqslant 2 \gamma_{1}\left[u_{0}\right] .
$$

Если же $t \in\left(1, T_{1}\right)$, то, используя, так же, как и в случае $\alpha+m<1$, леммы 2.1 и 2.2 , получаем

$$
\begin{aligned}
h(\rho) f_{B_{\rho / 2}} u(x, t) d x \leqslant & {\left[u_{0}\right]+\gamma C_{0}^{-\frac{m}{(m+1)^{2}}}\langle u\rangle_{t} } \\
& +\gamma C_{0}^{-\frac{N(p-1)}{m+1}} \int_{1}^{t} \frac{\langle u\rangle_{\tau}^{p} d \tau}{h^{p-1}\left(\mathscr{R}\left(C_{0} \tau\right)\right)} .
\end{aligned}
$$

Откуда в силу того, что $C_{0}$ можем выбрать достаточно большим, имеем

$$
\langle u\rangle_{t} \leqslant \gamma\left[u_{0}\right]+\gamma C_{0}^{\frac{N(p-1)}{m+1}-1} \int_{1}^{t} \frac{\langle u\rangle_{\tau}^{p} d \tau}{h^{p-1}(\mathscr{R}(\tau))} .
$$

Заметим, что $\langle u\rangle_{t}$ оценивается сверху функцией $y(t)$, которая является решением задачи

$$
\begin{gathered}
y^{\prime}=\gamma C_{0}^{\frac{N(p-1)}{m+1}-1} \frac{y^{p}}{h^{p-1}(\mathscr{R}(t))}, \quad 1<t<T_{1}, \\
y(1)=\gamma\left[u_{0}\right] .
\end{gathered}
$$


То есть функцией

$$
y(t)=y(1)\left(1-\gamma C_{0}^{\frac{N(p-1)}{m+1}-1}\left[u_{0}\right]^{p-1} \int_{1}^{t} \frac{d \tau}{h^{p-1}(\mathscr{R}(\tau))}\right)^{-\frac{1}{p-1}} .
$$

Очевидно, что $y(t) \leqslant \gamma y(1)$ при достаточно малом $\delta>0$. Отсюда имеем

$$
\langle u\rangle_{t} \leqslant \gamma\left[u_{0}\right], \quad 1<t<T_{1} .
$$

Покажем, наконец, что $T_{1}=\infty$. Как и ранее, применяя лемму 2.1 и неравенство (2.34), при всех $t \in\left(1, T_{1}\right)$ имеем

$$
t\|u(\cdot, t)\|_{\infty, \mathbb{R}^{N}}^{p-1} \leqslant \gamma\left[u_{0}\right]^{p-1} D_{0}+\left(\frac{\left[u_{0}\right]}{h(\mathscr{R}(1))}\right)^{p-1}<\frac{1}{4}
$$

для достаточно малых $\delta$. Из этого неравенства и определения $T_{1}$ следует, что $T_{1}=\infty$. Тогда из леммы 2.1 и оценки (2.34) заключаем, что для $t>0$ выполнена оценка (1.7). Теорема 1.1 доказана полностью.

ЛЕмма 2.3. Пусть и $(x, t)$ является непрерывныц решением уравнения (2.1) и $T>0$ такое, ито для всех $t, 0<t<T$, при $\rho>\mathscr{R}(C t)$ выполнено условие

$$
\frac{t}{\rho^{m+1}}\|u(\cdot, t)\|_{\infty, B_{2 \rho}\left(x_{0}\right)}^{m+\alpha-1}+t\|u(\cdot, t)\|_{\infty, \mathbb{R}^{N}}^{p-1}<1
$$

Тогда найдется постоянная $\gamma=\gamma(N, m, \alpha, p)$ такая, что при любъх $x_{0} \in \mathbb{R}^{N}$, $q_{1} \geqslant 1$ u $0<t<T$ верно неравенство

$$
\|u(\cdot, t)\|_{\infty, B_{\rho}\left(x_{0}\right) \times(t / 2, t)} \leqslant \gamma t^{-\frac{N}{K_{q_{1}}}}\left(f_{t / 4}^{t} \int_{B_{2 \rho}\left(x_{0}\right)} u^{q_{1}} d x d \tau\right)^{\frac{m+1}{K_{q_{1}}}} .
$$

ДоКАЗАТЕЛЬСТво ЛЕммы 2.3. Пусть $\sigma \in(0,1)$. Введем последовательности чисел $\rho_{n}=\rho\left(1+\sigma 2^{-n}\right), t_{n}=t\left(1-\sigma 2^{-n}\right) / 2$ и $k_{n}=k\left(1-2^{-(n+1)}\right), n=0,1,2, \ldots$, $k>0$ будет определено позже. Обозначим $B_{n} \equiv B_{\rho_{n}}\left(x_{0}\right), Q_{n} \equiv B_{n} \times\left(t_{n}, t\right)$. В частности, $Q_{0}=B_{\rho(1+\sigma)}\left(x_{0}\right) \times(t(1-\sigma) / 2, t), Q_{\infty}=B_{\rho} \times(t / 2, t)$. Определим также последовательность гладких срезающих функций $\varsigma_{n}=\varsigma_{n}(x, t)$ таких, что $0 \leqslant \varsigma_{n} \leqslant 1, \varsigma_{n}=1$ на $Q_{n+1}$ и $\varsigma_{n}=0$ вне $Q_{n}, 0 \leqslant\left(\varsigma_{n}\right)_{t} \leqslant 2^{n+2} /(\sigma t),\left|D \varsigma_{n}\right| \leqslant$ $2^{n+1} /(\sigma \rho)$. Введем функцию $\varphi_{n}=\left(v-k_{n}\right)_{+}^{q} \varsigma_{n}^{m+1}$, где $v$ то же, что и в лемме 2.2 , $q=q_{1} m /(\alpha+m)+1$ и $\left(v-k_{n}\right)_{+}=\max \left\{0, v-k_{n}\right\}$.

Домножив уравнение $(2.1)$ на $\varphi_{n}$ и проинтегрировав полученное уравнение по $Q_{n}$, получим

$$
\begin{aligned}
\iint_{Q_{n}} & \left(v^{\frac{m}{\alpha+m}}\right)_{t}\left(v-k_{n}\right)_{+}^{q} \varsigma_{n}^{m+1} d x d \tau \\
\leqslant & -\frac{m}{\alpha+m} \iint_{Q_{n}}\left(v-k_{n}\right)_{+}^{q-1}|D v|^{m+1} \varsigma_{n}^{m+1} d x d \tau \\
& +\frac{m(m+1)}{\alpha+m} \iint_{Q_{n}}\left(v-k_{n}\right)_{+}^{q}|D v|^{m} \varsigma_{n}^{m}\left|D \varsigma_{n}\right| d x d \tau \\
& +\iint_{Q_{n}} v^{\frac{m p}{\alpha+m}}\left(v-k_{n}\right)_{+}^{q} \varsigma_{n}^{m+1} d x d \tau
\end{aligned}
$$


Используя в левой части последнего выражения формулу интегрирования по частям и применяя неравенство Юнга во втором интеграле правой части с достаточно малой постоянной, имеем

$$
\begin{aligned}
\int_{B_{n}} \Lambda(x, t) \varsigma_{n}^{m+1} d x-\int_{B_{n}} \Lambda\left(x, t_{n}\right) \varsigma_{n}^{m+1} d x-(m+1) \iint_{Q_{n}} \Lambda(x, \tau) \varsigma_{n}^{m}\left(\varsigma_{n}\right)_{t} d x d \tau \\
\leqslant-\gamma \iint_{Q_{n}}\left(v-k_{n}\right)_{+}^{q-1}|D v|^{m+1} \varsigma_{n}^{m+1} d x d \tau \\
\quad+\gamma \iint_{Q_{n}}\left(v-k_{n}\right)_{+}^{q+m}\left|D \varsigma_{n}\right|^{m+1} d x d \tau+\iint_{Q_{n}} v^{\frac{m p}{\alpha+m}}\left(v-k_{n}\right)_{+}^{q} \varsigma_{n}^{m+1} d x d \tau
\end{aligned}
$$

где

$$
\Lambda(x, t)=\int_{k_{n}^{\alpha+m}}^{v^{\frac{m}{\alpha+m}}}\left(s^{\frac{\alpha+m}{m}}-k_{n}\right)_{+}^{q} d s=\frac{m}{\alpha+m} \int_{k_{n}}^{v} s^{-\frac{\alpha}{\alpha+m}}\left(s-k_{n}\right)_{+}^{q} d s .
$$

Из определения $\Lambda(x, t)$ следуют неравенства

$$
\begin{array}{rlr}
\Lambda(x, t) & \geqslant \frac{m}{(\alpha+m)(q+1)} k_{n}^{-\frac{\alpha}{\alpha+m}}\left(v-k_{n}\right)_{+}^{q+1} & \\
& \geqslant \frac{m 2^{\frac{\alpha}{\alpha+m}}}{(\alpha+m)(q+1)} k^{-\frac{\alpha}{\alpha+m}}\left(v-k_{n}\right)_{+}^{q+1}, \quad \alpha \leqslant 0, \\
\Lambda(x, t) & \geqslant \frac{m}{\alpha+m} v^{-\frac{\alpha}{\alpha+m}}\left(v-k_{n}\right)_{+}^{q+1}, & \alpha>0, \\
\Lambda(x, t) & \leqslant \frac{m}{\alpha+m}\left(v-k_{n}\right)_{+}^{q-1} \int_{k_{n}}^{v} s^{1-\frac{\alpha}{\alpha+m}} d s & \\
\leqslant \frac{m}{\alpha+2 m}\|v\|_{\infty, Q_{0}}^{\frac{\alpha+2 m}{\alpha+m}}\left(v-k_{n}\right)_{+}^{q-1} . &
\end{array}
$$

Рассмотрим сначала случай $\alpha \leqslant 0$. Из (2.36), (2.37.a), (2.38) следует оценка

$$
\begin{aligned}
k^{-\frac{\alpha}{\alpha+m}} & \sup _{t_{n} \leqslant \tau \leqslant t} \int_{B_{n}}\left(v-k_{n}\right)_{+}^{q+1} \varsigma_{n}^{m+1} d x \\
& +\iint_{Q_{n}}\left(v-k_{n}\right)_{+}^{q-1}|D v|^{m+1} \varsigma_{n}^{m+1} d x d \tau \\
\leqslant & \gamma \frac{2^{n+2}}{\sigma t}\|v\|_{\infty, Q_{0}}^{\frac{\alpha+2 m}{\alpha+m}} \iint_{Q_{n}}\left(v-k_{n}\right)_{+}^{q-1} d x d \tau \\
& +\gamma \frac{2^{n(m+1)}}{\sigma^{m+1} \rho^{m+1}}\|v\|_{\infty, Q_{0}}^{m+1} \iint_{Q_{n}}\left(v-k_{n}\right)_{+}^{q-1} d x d \tau \\
& +\gamma\|v\|_{\infty, Q_{0}}^{\frac{m p}{\alpha+m}+1} \iint_{Q_{n}}\left(v-k_{n}\right)_{+}^{q-1} d x d \tau .
\end{aligned}
$$

Пусть $\theta>\max \{1,(q+m) /(q+1)\}-$ фиксированное число и

$$
s=\frac{(m+1)(q+1)}{q+m}, \quad s^{\prime}=\frac{(m+1)(q-1)}{q+m} .
$$


Введем также функцию $\omega_{n}=\left(v-k_{n+1}\right)_{+}^{\frac{q+m}{m+1}} \varsigma_{n}^{\theta}$, тогда

$$
\begin{gathered}
\iint_{Q_{n}}\left(v-k_{n}\right)_{+}^{q-1}|D v|^{m+1} \varsigma_{n}^{m+1} d x d \tau \geqslant \gamma \iint_{Q_{n}}\left|D \omega_{n}\right|^{m+1} d x d \tau \\
-\gamma \frac{2^{n(m+1)}\|v\|_{\infty, Q_{0}}^{m+1}}{(\sigma \rho)^{m+1}} \iint_{Q_{n}}\left(v-k_{n}\right)_{+}^{q-1} d x d \tau
\end{gathered}
$$

и

$$
\begin{aligned}
& \sup _{t_{n} \leqslant \tau \leqslant t} \int_{B_{n}}\left(v-k_{n}\right)_{+}^{q+1} \varsigma_{n}^{m+1} d x \\
& \quad \geqslant \sup _{t_{n} \leqslant \tau \leqslant t} \int_{B_{n}}\left(v-k_{n+1}\right)_{+}^{q+1} \varsigma_{n}^{\frac{\theta(m+1)(q+1)}{q+m}} d x=\sup _{t_{n} \leqslant \tau \leqslant t} \int_{B_{n}} \omega_{n}^{s} d x .
\end{aligned}
$$

Из (2.39)-(2.41) имеем

$$
\begin{aligned}
& k^{-\frac{\alpha}{\alpha+m}} \sup _{t_{n} \leqslant \tau \leqslant t} \int_{B_{n}} \omega_{n}^{s} d x+\iint_{Q_{n}}\left|D \omega_{n}\right|^{m+1} d x d \tau \\
& \leqslant \gamma 2^{n(m+1)} \sigma^{-(m+1)}\|v\|_{\infty, Q_{0}}^{m+1}\left(\|v\|_{\infty, Q_{0}}^{\frac{m}{\alpha+m}(1-m-\alpha)} t^{-1}+\rho^{-(m+1)}\right. \\
& \left.\quad+\|v\|_{\infty, Q_{0}}^{\frac{m}{\alpha+m}(p-m-\alpha)}\right) \iint_{Q_{n}}\left(v-k_{n}\right)_{+}^{q-1} d x d \tau .
\end{aligned}
$$

В силу условий леммы из последнего неравенства получим

$$
\begin{gathered}
k^{-\frac{\alpha}{\alpha+m}} \sup _{t_{n} \leqslant \tau \leqslant t} \int_{B_{n}} \omega_{n}^{s} d x+\iint_{Q_{n}}\left|D \omega_{n}\right|^{m+1} d x d \tau \\
\leqslant \gamma 2^{n(m+1)} M \iint_{Q_{n}}\left(v-k_{n}\right)_{+}^{q-1} d x d \tau,
\end{gathered}
$$

здесь $M=\|v\|_{\infty, Q_{0}}^{\frac{m}{\alpha+m}(1-m-\alpha)+m+1} /\left(\sigma^{m+1} t\right)$.

Поскольку $\omega_{n}$ финитна на $Q_{n}$, то мы можем применить неравенство Гальярдо-Ниренберга, которое дает оценку

$$
\iint_{Q_{n}} \omega_{n}^{q^{\prime}} d x d \tau \leqslant \gamma\left(\iint_{Q_{n}}\left|D \omega_{n}\right|^{m+1} d x d \tau\right)\left(\sup _{t_{n} \leqslant \tau \leqslant t} \int_{B_{n}} \omega_{n}^{s} d x\right)^{\frac{m+1}{N}},
$$

где

$$
q^{\prime}=(m+1)\left(1+\frac{s}{N}\right)
$$

Поскольку $s^{\prime}<q^{\prime}$, то воспользуемся неравенством Гёльдера:

$$
\begin{aligned}
& \iint_{Q_{n+1}}\left(v-k_{n+1}\right)_{+}^{q-1} d x d \tau \leqslant \iint_{Q_{n}} \omega_{n}^{s^{\prime}} d x d \tau \\
& \leqslant\left(\iint_{Q_{n}} \omega_{n}^{q^{\prime}} d x d \tau\right)^{\frac{s^{\prime}}{q^{\prime}}}\left(\operatorname{mes}_{N} A_{n+1}\right)^{1-\frac{s^{\prime}}{q^{\prime}}},
\end{aligned}
$$

где $A_{n+1}=\left\{(x, t) \in Q_{n} \cap\left\{v>k_{n+1}\right\}\right\}$. 
Комбинируя (2.44) и (2.43) и оценивая каждый из сомножителей правой части (2.43) по неравенству (2.42), получаем

$$
\begin{aligned}
& \iint_{Q_{n+1}}\left(v-k_{n+1}\right)_{+}^{q-1} d x d \tau \leqslant \gamma k^{\frac{\alpha(m+1) s^{\prime}}{(\alpha+m) N q^{\prime}}}\left(\operatorname{mes}_{N} A_{n+1}\right)^{1-\frac{s^{\prime}}{q^{\prime}}} \\
& \quad \times\left(2^{n(m+1)} M \iint_{Q_{n}}\left(v-k_{n}\right)_{+}^{q-1} d x d \tau\right)^{\frac{(N+m+1) s^{\prime}}{N q^{\prime}}} .
\end{aligned}
$$

Оценим $\operatorname{mes}_{N} A_{n+1}$ следуюшим образом:

$$
\iint_{Q_{n} \cap\left\{v>k_{n+1}\right\}}\left(v-k_{n}\right)_{+}^{q-1} d x d \tau \geqslant\left(k_{n+1}-k_{n}\right)^{q-1}\left(\operatorname{mes}_{N} A_{n+1}\right) .
$$

Отсюда следует, что

$$
\operatorname{mes}_{N} A_{n+1} \leqslant k^{1-q_{2}}{ }^{(n+2)(q-1)} \iint_{Q_{n}}\left(v-k_{n}\right)_{+}^{q-1} d x d \tau
$$

Обозначим теперь $Y_{n}=\iint_{Q_{n}}\left(v-k_{n}\right)_{+}^{q-1} d x d \tau$. Тогда оценка $(2.45)$ с учетом (2.46) приводит нас к неравенству

$$
Y_{n+1} \leqslant \gamma b^{n} k^{-\left(\frac{(q-1)\left(q^{\prime}-s^{\prime}\right)}{q^{\prime}}-\frac{\alpha(m+1) s^{\prime}}{(\alpha+m) N q^{\prime}}\right)} M^{\frac{(N+m+1) s^{\prime}}{N q^{\prime}}} Y_{n}^{1+\frac{(m+1) s^{\prime}}{N q^{\prime}}},
$$

где

$$
b=2^{(m+1) \frac{(N+m+1) s^{\prime}}{N q^{\prime}}+(q-1)\left(1-\frac{s^{\prime}}{q^{\prime}}\right)}>1 .
$$

Если мы положим теперь

$$
k=\gamma M^{\frac{N+m+1}{m+1} r} Y_{0}^{r}, \quad r=\frac{\alpha+m}{(N+q)(\alpha+m)+m},
$$

то в силу итеративной леммы (см. [22; гл. II, лемма 5.6]) имеем

$$
\|v\|_{\infty, Q_{\infty}} \leqslant k
$$

или

$$
\begin{aligned}
\|v\|_{\infty, Q_{\infty}} & \leqslant \gamma\left(\frac{\|v\|_{\infty, Q_{0}}^{\frac{m}{\alpha+m}(1-m-\alpha)+m+1}}{\sigma^{m+1} t}\right)^{\frac{N+m+1}{m+1} r}\left(\iint_{Q_{0}}\left(v-\frac{k}{2}\right)_{+}^{q-1} d x d \tau\right)^{r} \\
& \leqslant \gamma\left(\frac{\|v\|_{\infty, Q_{0}}^{\frac{m}{\alpha+m}(1-m-\alpha)+m+1}}{\sigma^{m+1} t}\right)^{\frac{N+m+1}{m+1} r}\left(\iint_{Q_{0}} v^{q-1} d x d \tau\right)^{r} \cdot
\end{aligned}
$$

Далее воспользуемся стандартным методом итерации по $\sigma$ (см. [2], [21]). Пусть $\sigma=\sum_{i=1}^{l} 2^{-(i+1)}$. Обозначим также $\widetilde{Q}_{l}=Q_{0}$ при соответствуюшем $\sigma$ и $X_{l}=$ $\|v\|_{\infty, \widetilde{Q}_{l}}$. 
Применяя неравенство (2.47) к паре цилиндров $\widetilde{Q}_{l}, \widetilde{Q}_{l+1}, \widetilde{Q}_{l} \subset \widetilde{Q}_{l+1}$, имеем

$$
X_{l} \leqslant \gamma X_{l+1}^{\frac{(\alpha+2 m)(N+m)(m+1)}{(\alpha+m)} r}\left(2^{l(N+m+1)} t^{-\frac{N}{m+1}-1} \iint_{\widetilde{Q}_{l+1}} v^{q-1} d x d \tau\right)^{r} .
$$

Воспользуемся в левой части последнего выражения неравенством Юнга с некоторой $\nu>0$, которую определим позже:

$$
\begin{aligned}
X_{l} \leqslant & \nu X_{l+1}+\gamma(m, \alpha, N, p, \nu) 2^{\frac{l(N+m+1)(\alpha+m)(m+1)}{m K_{q_{1}}}} \\
& \times\left(t^{-\frac{N}{m+1}-1} \iint_{\widetilde{Q}_{l+1}} v^{q-1} d x d \tau\right)^{\frac{(\alpha+m)(m+1)}{m K_{q_{1}}}}
\end{aligned}
$$

(напомним, что $\left.q_{1}=(q-1)(\alpha+m) / m\right)$. Итерируя последнее неравенство, приходим к следуюшему соотношению:

$$
\begin{aligned}
X_{0} \leqslant & \nu^{l} X_{l}+\gamma(m, \alpha, N, p, \nu)\left(t^{-\frac{N}{m+1}-1} \iint_{\widetilde{Q}_{l+1}} v^{q-1} d x d \tau\right)^{\frac{(\alpha+m)(m+1)}{m K_{q_{1}}}} \\
& \times \sum_{i=1}^{l}\left(\nu 2^{\frac{(N+m+1)(\alpha+m)(m+1)}{m K_{q_{1}}}}\right)^{i-1}
\end{aligned}
$$

Выбираем теперь $\nu=2^{-\frac{(N+m+1)(\alpha+m)(m+1)}{m K_{q_{1}}}-1}$ и переходим в $(2.48)$ к пределу при $l \rightarrow \infty$. Это дает

$$
\|v\|_{\infty,\left(B_{\rho} \times(t / 2, t)\right)} \leqslant \gamma\left(t^{-\frac{N}{m+1}} f_{t / 4}^{t} \int_{B_{2 \rho}} v^{q-1} d x d \tau\right)^{\frac{(m+1)(\alpha+m)}{m K_{1}}} .
$$

Отсюда немедленно следует требуемое неравенство (2.35) при $\alpha \leqslant 0$. Пусть теперь $\alpha>0$. Из (2.36), (2.37.b), (2.38), (2.40), (2.41) имеем

$$
\begin{aligned}
& \|v\|_{\infty, Q_{0}}^{-\frac{\alpha}{\alpha+m}} \sup _{t_{n} \leqslant \tau \leqslant t} \int_{B_{n}} \omega_{n}^{s} d x+\iint_{Q_{n}}\left|D \omega_{n}\right|^{m+1} d x d \tau \\
& \quad \leqslant \gamma 2^{n(m+1)}\|v\|_{\infty, Q_{0}}^{\frac{\alpha+2 m}{\alpha+m}} \sigma^{-(m+1)} t^{-1} \iint_{Q_{n}}\left(v-k_{n}\right)_{+}^{q-1} d x d \tau .
\end{aligned}
$$

Правая часть отлична от нуля лишш при $v>k_{n}$, тогда

$$
\|v\|_{\infty, Q_{0}}^{-\frac{\alpha}{\alpha+m}} \leqslant k_{n}^{-\frac{\alpha}{\alpha+m}}
$$

и вместо (2.42) в этом случае мы получим

$$
\begin{gathered}
\|v\|_{\infty, Q_{0}}^{-\frac{\alpha}{\alpha+m}} \sup _{t_{n} \leqslant \tau \leqslant t} \int_{B_{n}} \omega_{n}^{s} d x+\iint_{Q_{n}}\left|D \omega_{n}\right|^{m+1} d x d \tau \\
\leqslant \gamma k^{-\frac{\alpha}{\alpha+m}} 2^{n(m+1)} \widetilde{M} \iint_{Q_{n}}\left(v-k_{n}\right)_{+}^{q-1} d x d \tau
\end{gathered}
$$


где $\widetilde{M}=\|v\|_{\infty, Q_{0}}^{2} /\left(\sigma^{m+1} t\right)$. Из неравенств $(2.43),(2.44)$ и $(2.50)$ следует, что

$$
\begin{aligned}
Y_{n+1} \leqslant & \gamma b^{n} k^{-\left(\frac{\alpha}{\alpha+m}\left(1+\frac{m+1}{N}\right) \frac{s^{\prime}}{q^{\prime}}+(q-1) \frac{q^{\prime}-s^{\prime}}{q^{\prime}}\right)} \\
& \times \widetilde{M}^{\left(1+\frac{m+1}{N}\right) \frac{s^{\prime}}{q^{\prime}}\|v\|_{\infty, Q_{0}}^{\frac{\alpha(m+1) s^{\prime}}{(\alpha+m) N q^{\prime}}} Y_{n}^{1+\frac{(m+1) s^{\prime}}{N q^{\prime}}}},
\end{aligned}
$$

здесь $Y_{n}$ и $b$ те же, что и в случае $\alpha \leqslant 0$.

Положим

$$
\begin{aligned}
& k=\widetilde{M} \frac{(N+m+1)(\alpha+m)}{\tilde{r}}\|v\|_{\infty, Q_{0}}^{\frac{\alpha(m+1)}{\tilde{r}}} Y_{0}^{\frac{(\alpha+m)(m+1)}{\tilde{r}}} \\
& \tilde{r}=\alpha(N+m+1)+(m+1)^{2}(\alpha+m)(N+q+1) .
\end{aligned}
$$

Из итеративной леммы (см. [22; гл. II, лемма 5.6]) следует оценка

$$
\|v\|_{\infty, Q_{\infty}} \leqslant \gamma \frac{\|v\|_{\infty, Q_{0}}^{\frac{2(\alpha+m)(N+m+1)+\alpha(m+1)}{\tilde{r}}}}{\left(\sigma^{m+1} t\right)^{\frac{(\alpha+m)(N+m+1)}{\tilde{r}}}}\left(\iint_{Q_{0}} v^{q-1} d x d \tau\right)^{\frac{(m+1)(\alpha+m)}{\tilde{r}}} .
$$

Из этого неравенства, повторяя ту же итеративную процедуру, что и в случае $\alpha \leqslant 0$, мы приходим к оценке (2.48). Лемма 2.3 доказана полностью.

Заметим теперь, что (2.8) переходит в (2.35) при $q_{1}=1$, а (2.24) следует немедленно из (2.35), поскольку (2.35) верно при любых $x_{0} \in \mathbb{R}^{N}$ и $\rho=\mathscr{R}(C t)$.

\section{§3. Доказательство теоремы 1.2}

В дальнейшем при доказательстве нам потребуется следующее утверждение.

Лемма 3.1. Пусть $\theta_{0}$ и $x_{0}$ mе жсе, что и в условии теоремы 1.2, а $\theta$, $0<\theta<\theta_{0}$, такое, что $m+\alpha-\theta>0$. Тогда при всех $t>0$ и $\rho \geqslant \mathscr{R}(C t)$ для решения $и(x, t)$ задачи (1.1), (1.2) имеет место следующая оценка:

$$
h^{1-\theta}(\rho) \int_{B_{2 \rho}} u^{1-\theta}(\cdot, t) d x \geqslant h^{1-\theta}(\rho) \int_{B_{2 \rho}} u_{0}^{1-\theta} d x-\gamma\left(h(\rho) \rho^{-\frac{m+1}{p-m-\alpha}}\right)^{m+\alpha-\theta}
$$

где $B_{2 \rho} \equiv B_{2 \rho}\left(x_{0}\right)$.

ДокАЗАТЕЛЬСТво. Умножим обе части уравнения (1.1) на пробную функцию $(u+\varepsilon)^{-\theta} \zeta^{s}(x)$, где $\varepsilon>0, s>m+1$, а $\zeta(x)$ - срезаюшая функция шара $B_{2 \rho}$ с теми же свойствами, что и в доказательстве леммы 2.2. Тогда получим

$$
\begin{aligned}
\frac{1}{1-\theta} & \int_{B_{2 \rho}} \zeta^{s}(u+\varepsilon)^{1-\theta} d x-\frac{1}{1-\theta} \int_{B_{2 \rho}} \zeta^{s}\left(u_{0}+\varepsilon\right)^{1-\theta} d x \\
= & \theta \int_{0}^{t} \int_{B_{2 \rho}} \zeta^{s}|D u|^{m+1} u^{\alpha}(u+\varepsilon)^{-\theta-1} d x d \tau \\
& -s \int_{0}^{t} \int_{B_{2 \rho}} \zeta^{s-1}|D u|^{m-1} u^{\alpha}(u+\varepsilon)^{-\theta} D u D \zeta d x d \tau \\
& +\int_{0}^{t} \int_{B_{2 \rho}} u^{p}(u+\varepsilon)^{-\theta} \zeta^{s} d x d \tau .
\end{aligned}
$$


Применяя неравенство Юнга ко второму слагаемому в правой части полученного равенства и устремляя $\varepsilon$ к нулю, получаем

$$
\begin{aligned}
\int_{B_{2 \rho}} \zeta^{s} u^{1-\theta}(\cdot, t) d x \geqslant & \int_{B_{2 \rho}} \zeta^{s} u_{0}^{1-\theta} d x-\frac{\gamma}{\rho^{m+1}} \int_{0}^{t} \int_{B_{2 \rho}} u^{\alpha+m-\theta} \zeta^{s-(m+1)} d x d \tau \\
& +\gamma \int_{0}^{t} \int_{B_{2 \rho}} u^{p-\theta} \zeta^{s} d x d \tau
\end{aligned}
$$

Еще раз применяя неравенство Юнга, имеем

$$
\begin{aligned}
& \frac{1}{\rho^{m+1}} \int_{0}^{t} \int_{B_{2 \rho}} u^{\alpha+m-\theta} \zeta^{s-(m+1)} d x d \tau \\
& \quad \leqslant \frac{\gamma}{2} \int_{0}^{t} \int_{B_{2 \rho}} u^{p-\theta} \zeta^{s} d x d \tau+\gamma t \rho^{N-\frac{(m+1)(p-\theta)}{p-m-\alpha}}
\end{aligned}
$$

при

$$
s=\frac{(m+1)(p-\theta)}{p-m-\alpha}>m+1 .
$$

Из полученного неравенства следует оценка

$$
\int_{B_{2 \rho}} \zeta^{s} u^{1-\theta}(\cdot, t) d x \geqslant \int_{B_{2 \rho}} \zeta^{s} u_{0}^{1-\theta} d x-\gamma t \rho^{N-\frac{(m+1)(p-\theta)}{p-m-\alpha}} .
$$

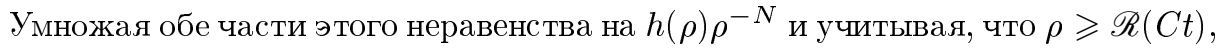
приходим к желаемому результату. Лемма 3.1 доказана.

Переходим к доказательству теоремы 1.2.

Поступая точно так же, как при доказательстве леммы 3.1 , из (3.2) и (3.3) получаем следующее неравенство:

$$
\frac{d}{d t} E(t) \geqslant-\gamma \rho^{N-\frac{(m+1)(p-\theta)}{p-m-\alpha}}+\gamma \int_{\mathbb{R}^{N}} u^{p-\theta} \zeta^{s} d x
$$

где $E(t)=\int_{B_{2 \rho}} \zeta^{s} u^{1-\theta} d x$, а $s$ определено в (3.4).

Предположим, что для всех $\rho>0$ выполнено неравенство

$$
\int_{\mathbb{R}^{N}} u^{p-\theta}(\cdot, t) \zeta^{s} d x \leqslant \gamma \rho^{N-\frac{(m+1)(p-\theta)}{p-m-\alpha}} .
$$

Тогда в силу неравенства Гёльдера для функции $E(t)$ справедлива оценка

$$
E(t) \leqslant \gamma\left(\int_{\mathbb{R}^{N}} u^{p-\theta} \zeta^{s} d x\right)^{\frac{1-\theta}{p-\theta}} \rho^{\frac{N(p-1)}{p-\theta}} \leqslant \gamma \rho^{N} \rho^{-\frac{(m+1)(1-\theta)}{p-m-\alpha}},
$$

или

$$
h^{1-\theta}(\rho) f_{B_{2 \rho}} u^{1-\theta} \zeta^{s} d x \leqslant \gamma\left(h(\rho) \rho^{-\frac{m+1}{p-m-\alpha}}\right)^{1-\theta}
$$


По условию теоремы правая часть стремится к нулю при $\rho \rightarrow \infty$. В то время как в силу леммы 3.1 левая часть оценивается снизу через

$$
h^{1-\theta}(\rho) f_{B_{2 \rho}} u_{0}^{1-\theta} d x-\gamma\left(h(\rho) \rho^{-\frac{m+1}{p-m-\alpha}}\right)^{m+\alpha-\theta}
$$

и, следовательно, ограничена снизу постоянной $C^{*}$, которая не равна нулю.

Таким образом, для достаточно больших $\rho$ имеет место неравенство

$$
\frac{d}{d t} E(t) \geqslant \gamma \int_{\mathbb{R}^{N}} u^{p-\theta} \zeta^{s} d x
$$

Неравенство Гёльдера дает

$$
E(t) \leqslant\left(\int_{\mathbb{R}^{N}} u^{p-\theta} \zeta^{s} d x\right)^{\frac{1-\theta}{p-\theta}}\left(\int_{\mathbb{R}^{N}} \zeta^{s} d x\right)^{\frac{p-1}{p-\theta}} .
$$

Следовательно, из (3.7) и последнего неравенства получаем оценку

$$
\frac{d}{d t} F(t) \geqslant F^{\frac{p-\theta}{1-\theta}}(t)
$$

где $F(t)=E(t) / \int_{\mathbb{R}^{N}} \zeta^{s} d x$.

Наконец, интегрируя неравенство (3.8), приходим к оценке

$$
F(t) \geqslant \frac{F(0)}{\left(1-\gamma F^{\frac{p-1}{1-\theta}}(0) t\right)^{\frac{1-\theta}{p-1}}} .
$$

Из этой оценки видно, что $F(t) \rightarrow \infty$ при $t \rightarrow T^{*}=\frac{1}{\gamma} F^{-\frac{p-1}{1-\theta}}(0)$.

Теорема 1.2 доказана.

\section{Список литературы}

1. Калашников A.C. Некоторые вопросы качественной теории нелинейных вырождающихся параболических уравнений второго порядка // УМН. 1987. Т. 42. №2(254). C. $135-176$.

2. Di Benedetto E., Herrero M. A. Non-negative solutions of the evolution $p$-Laplacian equation. Initial traces and Cauchy problem when $1<p<2 / /$ Arch. Ration. Mech. Anal. 1990. V. 111. P. 225-290.

3. Vespri $V$. On the local behavior of solutions of a certain class of doubly nonlinear parabolic equations // Manuscripta Math. 1992. V. 75. P. 65-80.

4. Tsutsumi $M$. On solution of some doubly nonlinear parabolic equations with absorbtion // J. Math. Anal. Appl. 1988. V. 132. P. 187-212.

5. Kaplan $S$. On the grouth of solutions of quasilinear parabolic equations // Comm. Pure Appl. Math. 1963. V. 16. P. 305-330.

6. Fujita $H$. On the blowing up of solutions to the Cauchy problem for $u_{t}=\Delta u+u^{1+\alpha} / /$ J. Fac. Sci. Univ. Tokyo Sect. I. 1966. V. 13. P. 109-124.

7. Hayakawa $K$. On nonexistence of global solutions of some semilinear parabolic differential equations // Proc. Japan Acad. Ser. A Math. Sci. 1973. V. 49. P. 503-505. 
8. Самарский А.А., Галактионов В.А., Курдюмов С.П., Михайлов А. П. Режимыс обострением в задачах для квазилинейных параболических уравнений. М.: Наука, 1987.

9. Galaktionov V.A., Levine H.A. A general approach to critical Fujita exponents and systems // Nonlinear Anal. 1998. V. 34. P. 1005-1027.

10. Andreucci D., Tedeev A.F. A Fujita type result for degenerate Neumann problem in domains with noncompact boundary // J. Math. Anal. Appl. 1999. V. 231. P. 543-567.

11. Andreucci D., Tedeev A.F. Optimal bounds and blow-up phenomena for parabolic problems in narrowing domains // Proc. Roy. Soc. Edinburg Sect. A. 1998. V. 128. №6. P. $1163-1180$.

12. Liu X., Wang $M$. The critical exponent of doubly singular parabolic equations // J. Math. Anal. Appl. 2001. V. 257. № 1. P. 170-188.

13. Mukai K., Mochuzuki K., Huang Q. Large time behavior and life span for a quasilinear parabolic equation with slow decay initial values // Nonlinear Anal. 2000. V. 39A. № 1 . P. 33-45.

14. Kamin S., Ughi $M$. On the behavior as $t \rightarrow \infty$ of the solutions the Cauchy problem for certain nonlinear parabolic equations // J. Math. Anal. Appl. 1987. V. 128. P. 456-469.

15. Deng K., Levine H. A. The role of critical exponents in blow-up theorems: The sequel // J. Math. Anal. Appl. 2000. V. 243. P. 85-126.

16. Levine H. A. The role of critical exponents in blow up theorems // SIAM Rev. 1990. V. 32. P. 262-288.

17. Cirmi G.R., Leonardi S., Tedeev A.F. The asymptotic behavior of the solution of a quasilinear parabolic equation with blow-up term // Preprint. Catania: Univ. Catania Dipart. Matem. Inform., 1998.

18. Andreucci D., Di Benedetto E. On the Cauchy problem and initial traces for a class of evolution equaions with strongly nonlinear sources // Ann. Scuola Norm. Sup. Pisa Cl. Sci. (4). 1991. V. 18. P. 363-441.

19. Andreucci $D$. Degenerate parabolic equations with initial data measares // Trans. Amer. Math. Soc. 1997. V. 340. № 10. P. 3911-3923.

20. Junning $Z$. On the Cauchy problem and initial traces for the evolution $p$-Laplacian equations with strongly nonlinear sources // J. Differential Equations. 1995. V. 121. P. 329-383.

21. Ishige $K$. On the existence of solutions of the Cauchy problem for a doubly nonlinear parabolic equation // SIAM J. Math. Anal. 1996. V. 27. № 5. P. 1235-1260.

22. Ладызсенская О. А., Солонников В. А., Уральцева Н. Н. Линейныеи квазилинейные уравнения параболического типа. М.: Наука, 1967.

Институт прикладной математики и механики

Поступила в редакцию

НАН Украины, г. Донецк

23.04 .2002 и 22.08 .2003

E-mail: nata@iamm.ac.donetsk.ua 